\title{
CONFORMAL MAPPING OF OPEN RIEMANN SURFACES $\left({ }^{1}\right)$
}

\author{
BY \\ ZEEV NEHARI
}

1. Introduction. It was recently shown by Ahlfors $[1]\left({ }^{2}\right)$ that the well known canonical conformal mapping of a schlicht domain of connectivity $n$ onto an $n$-times covered circle $[5,7]$ can be generalized, in the case of an open Riemann surface, in the following manner: An open Riemann surface of genus $g$ which is bounded by $n$ closed curves can be mapped conformally onto a multiply-covered circle, the number of coverings not exceeding $n+2 g$.

This result suggests to look for other types of canonical mappings of open Riemann surfaces which are analogous to the corresponding classical canonical mappings used in the case of multiply-connected schlicht domains. It will be shown in this paper that these mappings exist and that, moreover, many of the relations between various canonical mapping functions and domain functions known in the schlicht case have their counterpart in the theory of open Riemann surfaces.

In the following, $R$ will denote an open Riemann surface of genus $g$ which is bounded by $n$ closed curves. $R$ will be assumed to possess a finite Dirichlet integral, so that the existence of the fundamental harmonic domain functions of $R$ is assured. A system of $g$ pairs of canonical cuts $\left(\alpha_{k}, \beta_{k}\right)$, $k=1,2, \cdots, g$, will transform $R$ into a domain of connectivity $n+1$, provided the $\alpha_{k}, \beta_{k}$ have no points in common with the boundary curves $b_{v}, \nu=1, \cdots, n$, of $R$.

Pursuing the analogy of Riemann's theory of the Abelian integrals, we shall not start by considering single-valued functions on $R$, but we shall instead define certain standardized types of multiple-valued domain functions out of which the single-valued functions we are interested in will later on be built. While it is clear that these domain functions will have to show a general resemblance to the Abelian integrals of the first, second, and third kind on a closed Riemann surface, there are many different possibilities of defining them. For the purposes of conformal mapping of $R$ onto standard domains, it seems that the most convenient method is to define these functions in such a way that if $R$ is mapped, in the Schottky sense [11], onto "half" a closed Riemann surface $S$ of genus $n+2 g-1$, these functions are transformed into the normal Abelian integrals on $S$. They will therefore be termed functions of the first, second, and third kind.

2. Functions of the first kind. The functions $w_{k}(z), k=1, \cdots, g$, of the first

Presented to the Society, December 29, 1949; received by the editors August 26, 1948.

(1) Paper done under contract with the Office of Naval Research N5ori. 76-16 NR 043046.

(2) Numbers in brackets refer to the references cited at the end of the paper. 
kind are defined as follows: $w_{k}(z)$ is free of singularities in $R$ and has no periods about the cuts $\alpha_{l}, l \neq k$. About $\alpha_{k}, w_{k}(z)$ has the period 1 and on the boundary $\Gamma$ of $R, w_{k}(z)$ is pure imaginary.

In order to prove the existence of the functions $w_{k}(z)$, we proceed as follows. We write $w_{k}(z)=\omega_{k}(z)+i \tilde{\omega}_{k}(z)$ and have thus to find a harmonic function $\omega_{k}$ which vanishes on $\Gamma$, has no periods about the $\alpha_{l}, l \neq k$, and has the period 1 about $\alpha_{k}$; moreover, its harmonic conjugate $\tilde{\omega}_{k}(z)$ is to be free of periods about the $\alpha_{l}, l=1, \cdots, g$.

If $g(z, \zeta)$ is the Green's function of $R$ with respect to the point, we consider the harmonic function

$$
u\left(z ; \alpha_{k}\right)=\int_{\alpha_{k}} \frac{\partial g(z, \zeta)}{\partial n_{\zeta}} d s_{\zeta}
$$

where the integration is performed along $\alpha_{k}$. The function $u\left(z ; \alpha_{k}\right)$ vanishes for $z \in \Gamma$, since $g(z ; \zeta) \equiv 0$ for $z \in \Gamma$. Since $u\left(z ; \alpha_{k}\right)$ does not vanish identically, it cannot be single-valued in $R$. It will, however, only have a period about the cut $\alpha_{k}$ and no periods about the other cuts. This follows from the observation that the period of the conjugate of the Green's function about $\alpha_{k}$ is not affected by the singularity of the Green's function completing a circuit along one of the other cuts. In the case of a circuit about $\alpha_{k}$, however, this argument is not valid any more, since the order of the integration along $\alpha_{k}$ and of the singularity performing a circuit about $\alpha_{k}$ cannot be interchanged. We now form the function

$$
v(z)=u\left(z ; \alpha_{k}\right)+\sum_{l=1}^{g} \lambda_{l} u\left(z ; \beta_{l}\right),
$$

where the $\lambda_{l}$ are real constants. $v(z)$ clearly vanishes on $\Gamma$, has no periods about $\alpha_{l}, l \neq k$, and has a nonvanishing period about $\alpha_{k}$. We now determine the constants $\lambda_{l}$ in such a way as to make the periods of the harmonic conjugate of $v(z)$ about $\alpha_{l}, l=1, \cdots, g$, vanish. This will be possible if the determinant of the periods of the harmonic conjugates $\tilde{u}\left(z ; \beta_{l}\right)$ of $u\left(z ; \beta_{l}\right)$ about the $\alpha_{l}$ does not vanish. This is indeed the case. For suppose it does vanish. Then there would exist a linear combination

$$
\eta(z)=\sum_{l=1}^{g} \mu_{l} u\left(z ; \beta_{l}\right)
$$

whose harmonic conjugate $\tilde{\eta}(z)$ is free of periods about the $\alpha_{l}$. By Green's formula, we have

$$
\iint_{R}\left(\eta_{x}^{2}+\eta_{y}^{2}\right) d x d y=\int_{\Gamma} \eta d \tilde{\eta}+\sum_{l=1}^{g} \int_{\alpha_{l}} \eta d \tilde{\eta}+\sum_{l=1}^{g} \int_{\beta_{l}} \eta d \tilde{\eta},
$$

where the integrals over $\alpha_{l}$ and $\beta_{l}$ are to be taken, in different directions, 
along both edges of these cuts. The integral over $\Gamma$ vanishes, since $\eta \equiv 0$ on $\Gamma$. The pairs of integrals over the $\beta_{l}$ cancel each other, since $\eta$ has no periods about $\alpha_{l}$ and has therefore the same value on both edges of $\beta_{l}$. Finally, the integrals over $\alpha_{l}$ vanish, since we may write

$$
\int_{\alpha_{l}} \eta d \tilde{\eta}=q_{l} \int_{\alpha_{l}} d \tilde{\eta}
$$

where $q_{l}$ is the period of $\eta$ about $\beta_{l}$ and the integration is to be extended over one edge of $\alpha_{l}$ only. Since $\tilde{\eta}$ has no periods about $\alpha_{l}$, the last integral vanishes. Hence

$$
\iint_{R}\left(\eta_{x}^{2}+\eta_{y}^{2}\right) d x d y=0
$$

whence $\eta \equiv 0$, since $\eta=0$ on $\Gamma$. But this is possible only if all the $\mu_{l}$ in (2) are zero, since otherwise $\eta$ would have nonvanishing periods about some of the $\beta_{l}$.

We have thus proved that the constants $\lambda_{l}$ in (1) can be so chosen as to make the periods of the conjugate of $v(z)$ about the $\alpha_{l}$ vanish. The particular function for which this is the case, and which has been multiplied by a suitable factor so as to make its period about $\alpha_{k}$ equal to 1 , will be denoted by $\omega_{k}(z)$. It is immediately confirmed that the analytic function

$$
w_{k}(z)=\omega_{k}(z)+i \tilde{\omega}_{k}(z),
$$

whose real part is $\omega_{k}(z)$, is the function of the first kind defined at the beginning of this section.

It is convenient to introduce yet another type of functions of the first kind, $w_{k}^{*}(z)$, which will be defined as follows: $w^{*}(z)$ is free of singularities in $R$ and has no periods about the cuts $\alpha_{l}, l \neq k$. About $\alpha_{k}, w_{k}^{*}(z)$ has the period 1 and on the boundary $\Gamma$ of $R, w_{k}^{*}(z)$ is real. The existence of the functions $w_{k}^{*}(z)$ is shown by a slight modification of the preceding argument.

There is yet a third type of functions which may also, with some justification, be called functions of the first kind. These functions, which we shall denote by $W_{\nu}(z), \nu=1, \cdots, n$, are defined as follows. If $\Omega_{\nu}(z)$ denotes the real part of $W_{\nu}(z)$, then $\Omega_{\nu}(z)$ is harmonic in $R$, vanishes on $\Gamma_{\mu}, \mu \neq \nu$, and takes the value 1 on $\Gamma_{\nu}$; moreover, $W_{\nu}(z)$ has no periods about the $\alpha_{k}, k=1, \cdots, g$. The function $\Omega_{\nu}(z)$, which is an immediate generalization of the usual harmonic measure, is easily constructed by completing the function

$$
\frac{1}{2 \pi} \int_{\Gamma_{\nu}} \frac{\partial g(z, \zeta)}{\partial n_{\zeta}} d s_{\zeta}
$$

to an analytic function and adding a suitable linear combination of functions $w_{k}^{*}(z)$ with imaginary coefficients so as to make its periods about the $\alpha_{k}$, $k=1, \cdots, g$, vanish. 
Only $n-1$ of the functions $\Omega_{\nu}(z)$ are linearly independent. Indeed, the function $\sum_{\nu=1}^{n} \Omega_{\nu}-1$ vanishes on $\Gamma$ and both this function and its conjugate have no periods about the $\alpha_{k}$. As shown above, such a function vanishes identically.

3. Functions of the second kind. We introduce two types of functions of the second kind, $t(z, \zeta)$ and $t^{*}(z, \zeta)$, by the following definitions: $t(z, \zeta)$ and $t^{*}(z, \zeta)$ are free of singularities in $R$, except for a simple pole with residue 1 at $z=\zeta(\zeta \in R)$. Both $t(z, \zeta)$ and $t^{*}(z, \zeta)$ have no periods about the $\alpha_{l}, l=1, \cdots$, $g$, and the boundary components $\Gamma_{1}, \cdots, \Gamma_{n} . \operatorname{Re}\left\{t^{*}(z, \zeta)\right\}$ and $\operatorname{Im}\{t(z, \zeta)\}$ are constant on each $\Gamma_{\nu}, \nu=1, \cdots, n$.

The existence of these functions is not difficult to show. We first find, by solving a Dirichlet problem, the harmonic functions

$$
h(z)=\operatorname{Re}\left\{\frac{1}{z-\zeta}\right\}+h_{1}(z), \quad k(z)=\operatorname{Im}\left\{\frac{1}{z-\zeta}\right\}+k_{1}(z),
$$

with $h_{1}(z)$ and $k_{1}(z)$ harmonic in $R$, which vanish on $\Gamma$. If $\sigma_{\nu}(z), \nu=1, \cdots, n$, denote the harmonic measures

$$
\sigma_{\nu}(z)=\frac{1}{2 \pi} \int_{\Gamma_{\nu}} \frac{\partial g(z, \zeta)}{\partial n_{\zeta}} d s_{\zeta}
$$

of the boundary components $\Gamma_{\nu}$, we construct linear combinations

$$
h_{2}(z)=h(z)+\sum_{\nu=1}^{n-1} \gamma_{\nu} \sigma_{\nu}(z), \quad k_{2}(z)=k(z)+\sum_{\nu=1}^{n-1} \gamma_{\nu}^{\prime} \sigma_{\nu}(z),
$$

whose harmonic conjugates are free of periods about the $\Gamma_{\nu}, \nu=1, \cdots, n$. This is possible for the same reasons as in the schlicht case.

If $\tilde{h}_{2}(z)$ and $\widetilde{k}_{2}(z)$ denote the harmonic conjugates of $h_{2}(z)$ and $k_{2}(z)$, respectively, the functions

$$
H_{2}(z)=h_{2}(z)+i \tilde{k}_{2}(z), \quad K_{2}(z)=\tilde{k}_{2}(z)-i k_{2}(z)
$$

will have constant real or imaginary parts, respectively, on $\Gamma_{\nu}, \nu=1, \cdots, n$, and will be free of periods about the $\Gamma_{\nu}$. The "interior" periods of $H_{2}(z)$ are imaginary and those of $K_{2}(z)$ are real. By adding a suitable linear combination with real coefficients of functions $i w_{k}^{*}(z)$ to $H_{2}(z)$ and of functions $w_{k}^{*}(z)$ to $K_{2}(z)$, the $g$ periods of these functions about the $\alpha_{l}, l=1, \cdots, g$, can be made to vanish. The possibility of finding these linear combinations is shown in exactly the same way as in a similar case in the previous section. It is then easily confirmed that the functions obtained by this process are indeed the functions of the second kind, $t^{*}(z, \zeta)$ and $t(z, \zeta)$, defined above.

4. Periods of the functions of the second kind. The periods of the functions of the second kind about the $\beta_{k}$ are expressible in terms of the functions of the first kind. We have 


$$
\begin{aligned}
& \frac{1}{2 \pi i} \int_{\beta_{k}} t^{* \prime}(z, \zeta) d z=\operatorname{Re}\left\{w_{k}^{\prime}(\zeta)\right\}+i \operatorname{Im}\left\{w_{k}^{* \prime}(\zeta)\right\} \\
& \frac{1}{2 \pi i} \int_{\beta_{k}} t^{* \prime}(z, \zeta) d z=\operatorname{Re}\left\{w_{k}^{* \prime}(\zeta)\right\}+i \operatorname{Im}\left\{w_{k}^{\prime}(\zeta)\right\} .
\end{aligned}
$$

These relations are analogous to those holding on a closed Riemann surface and, like those, are most easily proved by Riemann's method of contour integration. Let $R^{*}$ denote the domain of connectivity $n+1$ into which $R$ is transformed by a system of canonical cuts $\left(\alpha_{k}, \beta_{k}\right), k=1, \cdots, g$, and $\Gamma^{*}$ its boundary. We then have, by the residue theorem,

$$
\frac{1}{2 \pi i} \int_{\Gamma^{*}} t^{*}(z, \zeta) w^{\prime}(z) d z=w_{k}^{\prime}(\zeta)
$$

The integration path $\Gamma^{*}$ is composed of the boundary components $\Gamma_{\nu}$, $\nu=1, \cdots, n$, and the closed curves $\alpha_{l}, \beta_{l}, l=1, \cdots, g$, the latter taken twice, in different directions. Along $\beta_{l}$, we have, since $t^{*}(z, \zeta)$ is free of periods about $\alpha_{l}$ and $t^{*}(z, \zeta)$ has therefore the same values on both edges of $\beta_{l}$,

$$
\int_{\beta_{l}(-)} t^{*}(z, \zeta) w_{k}^{\prime}(z) d z+\int_{\beta_{l}(+)} t^{*}(z, \zeta) w_{k}^{\prime}(z) d z=0 .
$$

Along $\alpha_{l}$ we have

$$
\begin{aligned}
\int_{\alpha_{l}(-)} t^{*}(z, \zeta) w_{k}^{\prime}(z) d z+\int_{\alpha_{l}(+)} t^{*}(z, \zeta) w_{k}^{\prime}(z) d z & \\
& =p_{l}(t) \int_{\alpha_{l}(-)} w_{k}^{\prime}(z) d z=p_{l}(t) \int_{\alpha_{l}} d w_{k}(z),
\end{aligned}
$$

where $p_{l}(t)$ is the period of $t^{*}(z, \zeta)$ about $\beta_{l}$. For $l \neq k$, the last integral vanishes and for $l=k$ it has the value 1 . Thus,

$$
w_{k}^{\prime}(\zeta)=\frac{1}{2 \pi i} \int_{\beta_{k}} t^{* \prime}(z, \zeta) d z+\sum_{\nu=1}^{n} \frac{1}{2 \pi i} \int_{\Gamma_{\nu}} t^{*}(z, \zeta) w_{k}^{\prime}(z) d z .
$$

The values of the integrals over $\Gamma_{\nu}$ are real. Indeed,

$$
\int_{\Gamma_{\nu}} t^{*}(z, \zeta) w_{k}^{\prime}(z) d z=-\int_{\Gamma_{\nu}} w_{k}(z) t^{* \prime}(z, \zeta) d z,
$$

since both $t^{*}(z, \zeta)$ and $w_{k}(z)$ have no periods about $\Gamma_{\nu} \cdot w_{k}(z)$ is pure imaginary on $\Gamma$ and the same is true of $t^{* \prime}(z, \zeta) d z=d t^{*}(z, \zeta)$. As a result, the integrals are real, and we have

$$
\operatorname{Re}\left\{\frac{1}{2 \pi i} \int_{\beta_{k}} t^{* \prime}(z, \zeta) d z\right\}=\operatorname{Re}\left\{w_{k}^{\prime}(z)\right\} .
$$


By the same procedure, we also obtain

$$
\operatorname{Im}\left\{\frac{1}{2 \pi i} \int_{\beta_{k}} t^{* \prime}(z, \zeta) d z\right\}=\operatorname{Im}\left\{w_{k}^{* \prime}(z)\right\} .
$$

Combining (5) and (6), we arrive at (3). (4) follows by the same argument, applied to $t(z, \zeta)$.

Before we pass to the functions of the third kind on $R$, we shall discuss two domain functions closely allied to $t(z, \zeta)$ and $t^{*}(z, \zeta)$.

5. The Bergman kernel function. On $\Gamma, d t^{*}(z, \zeta)$ is pure imaginary and $d t(z, \zeta)$ is real. For the same reasons as in the case of schlicht domains $[6,8]$, it will therefore be useful to introduce the functions

$$
M(z)=M(z, \zeta)=2^{-1}\left[t(z, \zeta)-t^{*}(z, \zeta)\right]
$$

and

$$
N(z)=N(z, \zeta)=2^{-1}\left[t(z, \zeta)+t^{*}(z, \zeta)\right],
$$

which, on $\Gamma$, are connected by the relation

$$
N^{\prime} d z=\overline{M^{\prime} d z} .
$$

$N$ clearly has a simple pole with the residue 1 at $z=\zeta$ and $M$ has no singularities in $R$, and both $M$ and $N$ have the same periodic behaviour in $R$ as $t$ and $t^{*}$.

In the schlicht case, $\pi M^{\prime}(z, \zeta)$ is identical with the Bergman kernel function $K(z, \bar{\zeta})[2,3,4]$ of the class of regular square-integrable functions with a single-valued integral. We shall now show that a similar result holds in the case of the open Riemann surface $R$.

Let $f(z)$ be a function which has no singularities in $R$, is continuous in $R+\Gamma$, and has no periods about the $\Gamma_{v}$ and $\alpha_{k}$, and consider the integral

$$
I=\frac{1}{2 \pi i} \int_{\Gamma^{*}} f(z) N^{\prime}(z, \zeta) d z,
$$

taken over the boundary $\Gamma^{*}$ of the domain $R^{*}$ into which $R$ is transformed by a system of canonical cuts. The integral exists, since the differential $N^{\prime} d z$ is regular on $\Gamma$ and the $\alpha_{k}, \beta_{k}$ can be chosen to be analytic curves. Since the integrand is single-valued in $R^{*}$, we have, by the residue theorem,

$$
I=-f^{\prime}(\zeta)
$$

The integrals over the $\alpha_{k}, \beta_{k}$ are to be taken over both edges of each cut, in different directions. Since both $f(z)$ and $N^{\prime}(z, \zeta)$ have no periods about $\alpha_{k}$, they have the same values on both edges of $\beta_{k}$. As a result, the integrals over $\beta_{k}$ cancel out. On both edges of $\alpha_{k}$, the values of $f(z)$ differ by the period of $f(z)$ over $\beta_{k}$, say $q_{k}$. Hence, 


$$
\int_{\alpha_{k}(-)} f N^{\prime} d z+\int_{\alpha_{k}(+)} f N^{\prime} d z=q_{k} \int_{\alpha_{k}} N^{\prime} d z,
$$

and this again vanishes, since $N$ has no periods about $\alpha_{k}$. We thus obtain

$$
I=-f^{\prime}(\zeta)=\frac{1}{2 \pi i} \int_{\Gamma} f(z) N^{\prime}(z, \zeta) d z .
$$

or, in view of (9),

$$
-\bar{I}=\overline{f^{\prime}(\zeta)}=\frac{1}{2 \pi i} \int_{\Gamma} \overline{f(z)} M^{\prime}(z, \zeta) d z .
$$

By employing the same procedure as before to the integrals $\left(\int_{\alpha_{k}(-)}\right.$ $\left.+\int_{\alpha_{k}(+)}\right) \bar{f} M^{\prime} d z$ and $\left(\int_{\beta_{k}(+)}+\int_{\beta_{k}(-)}\right) \bar{f} M^{\prime} d z$, we see that these integrals also vanish. (10) may therefore be replaced by

$$
\overline{f^{\prime}(\zeta)}=\frac{1}{2 \pi i} \int_{\Gamma^{*}} \overline{f(z)} M^{\prime}(z, \zeta) d z,
$$

where the integration is now extended, in the positive sense, along the whole boundary of $R^{*}$. Since both $f$ and $M$ are single-valued and regular in $R^{*}$, we may use Green's formula in order to transform the line integral on the righthand side of (11) into an area integral. We obtain

$$
\overline{f^{\prime}(\zeta)}=\frac{1}{\pi} \iint_{R^{*}} \overline{f^{\prime}(z)} M^{\prime}(z, \zeta) d x d y
$$

or

$$
f^{\prime}(\zeta)=\frac{1}{\pi} \iint_{R} \overline{M^{\prime}(z, \zeta) f^{\prime}}(z) d x d y,
$$

where $R^{*}$ has been replaced by $R$, as this makes no difference in the area integration. Although (12) has so far only been proved for functions $f(z)$ which are continuous in $R+\Gamma$, this relation will, by a standard argument, also hold for functions which are not continuous on $\Gamma$.

(12) shows that the function

$$
K(z, \bar{\zeta})=\frac{1}{\pi} M^{\prime}(z, \zeta)
$$

has the characteristic reproducing property of the Bergman kernel function with regard to the class $\mathcal{L}^{2}(R)$ of all regular and single-valued functions $f^{\prime}(z)$ in $R$ which are square-integrable over $R$ and whose integrals are free of periods about the $\Gamma_{\nu}$ and $\alpha_{k}$. Since $K(z, \bar{\zeta})$ is also in $\mathcal{L}^{2}(R)$, it is therefore identical with the kernel function. By the well known formalism of the kernel 
function $[2,3,4], K(z, \bar{\zeta})$ may be computed-if the area of $R$ is finite-in terms of a complete system $\left\{\phi_{\mu}(z)\right\}$ of functions in $\mathcal{L}^{2}(R)$ which are orthonormalized by the conditions

$$
\iint_{R} \phi_{\mu}(z) \overline{\phi_{\tau}(z)} d x d y=\delta_{\mu \tau}
$$

We have

$$
K(z, \bar{\zeta})=\sum_{\mu=1}^{\infty} \phi_{\mu}(z) \overline{\phi_{\mu}(\zeta)}
$$

where the series on the right-hand side converges absolutely and uniformly in every closed subdomain of $R$. We omit the proof of the expansion (14) and its convergence, since it is word for word the same as in the case of a schlicht domain. If the area of $R$ is infinite, we shall obtain the kernel function by the formula (14), if we restrict the class $\mathcal{L}^{2}(B)$ to contain only such functions which have a double zero at those points of $R$ which lie above $z=\infty$.

Since the kernel function algorithm (14) seems to be the only computational approach to the domain functions on $R$ which has any degree of practical feasibility, it is of interest to express both $t(z, \zeta)$ and $t^{*}(z, \zeta)$-and not only their difference-in terms of the kernel function. Generalizing the procedure leading to a similar formula in the schlicht case [9], this can be done as follows. Let $v(z, \eta)$ be any function which has no singularities in $R+\Gamma$ except a simple pole with residue 1 at $z=\eta$, and which has no periods about the $\Gamma_{\nu}$ and the $\alpha_{k}$; we may, for instance, take $v(z, \eta)$ to be the normal Abelian integral of the second kind on the smallest closed Riemann surface of genus $g$ into which $R$ can be embedded. Consider the integral

$$
I=\frac{1}{2 \pi i} \int_{\Gamma^{*}} 2^{-1}\left[t^{\prime}(\eta, \zeta)+t^{* \prime}(\eta, \zeta)\right] v(\eta, z) d \eta
$$

where $\Gamma^{*}$ again denotes the boundary of $R^{*}$. By the residue theorem, we have

$$
I=2^{-1}\left[t^{\prime}(z, \zeta)+t^{* \prime}(z, \zeta)\right]-v^{\prime}(\zeta, z) .
$$

As before, it is easily seen that the integrals over $\Gamma^{*}-\Gamma$ cancel out, and we may therefore also write

$$
I=\frac{1}{2 \pi i} \int_{\Gamma} 2^{-1}\left[t^{\prime}(\eta, \zeta)+t^{* \prime}(\eta, \zeta)\right] v(\eta, z) d \eta
$$

or, by (8), (9), and (13),

$$
I=\frac{1}{2 i} \int_{\Gamma} \overline{K(\eta, \bar{\zeta})} v(\eta, z) \overline{d \eta} .
$$


Since, by (14),

$$
\overline{K(\eta, \bar{\zeta})}=K(\zeta, \bar{\eta})
$$

this may also be written

$$
I=\frac{1}{2 i} \int_{\mathrm{r}} K(\zeta, \bar{\eta}) v(\eta, z) \overline{d \eta}
$$

Combining this with (7), (13), and (15), we finally obtain

$$
\begin{aligned}
& t^{\prime}(z, \zeta)=v^{\prime}(\zeta, z)+K(z, \bar{\zeta})+\frac{1}{2 i} \int_{\Gamma} v(\eta, z) K(\zeta, \bar{\eta}) \overline{d \eta} \\
& t^{* \prime}(z, \zeta)=v^{\prime}(\zeta, z)-K(z, \xi)+\frac{1}{2 i} \int_{\Gamma} v(\eta, z) K(\zeta, \bar{\eta}) \overline{d \eta}
\end{aligned}
$$

These are the desired formulas for $t^{\prime}(z, \zeta)$ and $t^{* \prime}(z, \zeta)$ in terms of the kernel function.

We add here a formula showing the relations between the kernel function and the functions of the first kind. Combining (3), (4), (7), and (13), and remembering that $K(z, \bar{\zeta})=\overline{K(\zeta, \bar{z})}$, we obtain

$$
\frac{1}{i} \int_{\beta_{k}} K(\zeta, \bar{z}) \overline{d z}=w_{k}^{\prime}(z)-w_{k}^{* \prime}(z) .
$$

The analogous formula for the function $N(z, \zeta)$ is

$$
\frac{1}{\pi i} \int_{\beta_{k}} N(z, \zeta) d z=w_{k}^{\prime}(z)+w_{k}^{* \prime}(z) .
$$

6. Parallel slit mappings. We shall now prove the following theorem:

Theorem. Given an open Riemann surface $R$ of genus $g$, and $g+1$ points $\zeta_{0}, \zeta_{1}, \cdots, \zeta_{0}$ on $R$, there are two possibilities:

(a) Either $R$ can be mapped conformally by a function $w=F(z)$ onto a $(g+1)$-times covered plane with slits parallel to the real axis such that $F\left(\zeta_{k}\right)=\infty$, $k=0,1, \cdots, g$, and the residue of $F(z)$ at $z=\zeta_{0}$ is 1 , or

(b) it is possible to map $R$ conformally onto a less than $(g+1)$-times covered plane with slits parallel to the real axis, such that the poles of the mapping function coincide with $g$ or less of the points $\zeta_{k}, k=1, \cdots, g$.

Proof. Consider the function

$$
F(z)=t\left(z, \zeta_{0}\right)+\sum_{k=1}^{g}\left[a_{k} t\left(z, \zeta_{k}\right)+i b_{k} t^{*}\left(z, \zeta_{k}\right)\right]
$$

where $a_{k}, b_{k}$ are real constants. From the definitions of $t$ and $t^{*}$ it is obvious that $\operatorname{Im}\{F(z)\}$ is constant on each $\Gamma_{\nu} . F(z)$ will therefore yield the required 
parallel slit mapping, if it is possible to choose the constants $a_{k}, b_{k}$ in such a way as to make $F(z)$ single-valued on $R$. Since the only possible periods of $F(z)$ are those about the $\beta_{k}$, the conditions to be imposed on the $a_{k}, b_{k}$ will read, in view of (3) and (4),

$$
\begin{aligned}
&-\operatorname{Re}\left\{w_{k}^{* \prime}\left(\zeta_{0}\right)\right\}= \sum_{l=1}^{g} a_{l} \operatorname{Re}\left\{w_{k}^{* \prime}\left(\zeta_{l}\right)\right\}-b_{l} \operatorname{Im}\left\{w_{k}^{* \prime}\left(\zeta_{l}\right)\right\}, \\
&-\operatorname{Im}\left\{w_{k}^{\prime}\left(\zeta_{0}\right)\right\}=\sum_{l=1}^{g} a_{l} \operatorname{Im}\left\{w_{k}^{\prime}\left(\zeta_{l}\right)\right\}+b_{l} \operatorname{Re}\left\{w_{k}^{\prime}\left(\zeta_{l}\right)\right\}, \\
& k=1,2, \cdots, g .
\end{aligned}
$$

This is a system of $2 g$ linear equations for the $2 g$ unknowns $a_{l}, b_{l}, l=1, \cdots, g$. If the determinant of this system does not vanish, it has a solution and we are in the case (a) of the theorem. If the determinant vanishes, there exists at least one nontrivial solution of the homogeneous system and it is easily seen that the alternative (b) of the theorem is realized. This completes the proof of the theorem.

There is yet another way of constructing parallel slit mappings. Instead of giving the location of the poles and then determining the residues in such a way that the periods vanish, we can give the residues and then try to determine the location of the poles in accordance with this condition. The problem to be solved may be stated as follows. Given $g$ complex constants $\gamma_{l}, l=1, \cdots, g\left[\gamma_{l}=a_{l}+i b_{l}\right.$ in (20)], and a point $\zeta_{0}$ on $R$, to find $g$ points $\zeta_{1}, \cdots, \zeta_{\theta}$ on $R$ such that the equations

$$
\begin{aligned}
& \operatorname{Re}\left\{w_{k}^{* \prime}\left(\zeta_{0}\right)+\sum_{l=1}^{g} \gamma_{l} w^{* \prime}\left(\zeta_{l}\right)\right\}=0, \\
& \operatorname{Im}\left\{w_{k}^{\prime}\left(\zeta_{0}\right)+\sum_{l=1}^{g} \gamma_{l} w^{\prime}\left(\zeta_{l}\right)\right\}=0, \quad k=1, \cdots, g,
\end{aligned}
$$

are satisfied.

The question as to the solvability of the system (21) is a highly transcendental problem allied to the well known Jacobi inversion problem $[10,12]$, and we shall not pursue it here any further.

7. Representation of the Ahlfors mapping in terms of the kernel function. We shall show in this section that the functions $w=G(z)$ which map $R$ onto the $(n+2 g)$-times covered unit circle can be expressed in terms of kernel functions. We have

$$
G^{\prime}(z)=\pi \sum_{i=1}^{n+2 g} \bar{\delta}_{i} K\left(z, \bar{z}_{i}\right),
$$

where $z_{i}$ are the zeros of $G(z)$ and $G^{\prime}\left(z_{i}\right)=\delta_{i}^{-1}$.

Let $f(z)$ be a function which has no singularities on $R$ and is free of 
periods about the $\alpha_{k}, k=1, \cdots, g$, and the $\Gamma_{\nu}, \nu=1, \cdots, n$. We further assume that $f^{\prime}(z)$ is continuous in $R+\Gamma$. Since $f^{\prime}(z)[G(z)]^{-1}$ is single-valued in $R$, we have, by the residue theorem,

$$
I=\frac{1}{2 \pi i} \int_{\Gamma} \frac{f^{\prime}(z)}{G(z)} d z=\sum_{i=1}^{n+2 \sigma} \delta_{i} f^{\prime}\left(z_{i}\right) .
$$

Since, on $\Gamma,|G(z)|=1$, we may also write

or, using Green's formula,

$$
I=\frac{1}{2 \pi i} \int f^{\prime}(z) \overline{G(z)} d z,
$$

$$
I=\frac{1}{\pi} \iint_{R} f^{\prime}(z) \overline{G^{\prime}(z)} d x d y .
$$

Clearly, we may now drop the condition that $f^{\prime}(z)$ is continuous on $\Gamma$. Combining (24) with (23), we have thus the formula

$$
\frac{1}{\pi} \iint_{R} f^{\prime}(z) \overline{G^{\prime}(z)} d x d y=\sum_{i=1}^{n+2 g} \delta_{i} f^{\prime}\left(z_{i}\right) .
$$

On the other hand, if $H(z)$ denotes the function

$$
H(z)=\pi \sum_{i=1}^{n+2 g} \bar{\delta}_{i} K\left(z, \overline{z_{i}}\right),
$$

where $K\left(z, z_{i}\right)$ is the Bergman kernel function, we have, by (12),

$$
\frac{1}{\pi} \iint_{R} f^{\prime}(z) \overline{H(z)} d x d y=\sum_{i=1}^{n+2 g} \delta_{i} f^{\prime}\left(z_{i}\right) .
$$

Comparing this with (25), we obtain

$$
\iint_{R} f^{\prime}(z) \overline{\left[G^{\prime}(z)\right.} \overline{-H(z)]} d x d y=0 .
$$

Since $\left[G^{\prime}(z)-H(z)\right]$ satisfies all the conditions imposed on $f^{\prime}(z)$, we may set, in (26), $f^{\prime}(z)=G^{\prime}(z)-H(z)$. We thus obtain

$$
\iint_{R}\left|G^{\prime}(z)-H(z)\right|^{2} d x d y=0
$$

whence

$$
G^{\prime}(z) \equiv H(z)
$$

This proves the identity (22).

It is worthy of note that in reality we did not use the assumption that $f(z)$ 
is free of periods about the $\alpha_{k}$ and the $\Gamma_{\nu}$. We therefore have the interesting result that the formula (22) remains true if our particular kernel $K(z, \bar{\zeta})$ is replaced by the kernel of the family of all single-valued square-integrable functions on $R$, or the kernel of any subclass of this class whose integral has an arbitrarily prescribed periodic behaviour on $R$.

8. Functions of the third kind. There are again two types of functions of the third kind. The first of these, to be denoted by $p(z, \zeta)$, is an obvious generalization of the functions of the second kind defined above. $p(z, \zeta)$ is regular in $R$, except at the point $z=\zeta$, where $p(z, \zeta)+\log (z-\zeta)$ is regular; the real part of $p(z, \zeta)$ is constant on the boundary components $\Gamma_{\nu}$, and $p(z, \zeta)$ has no periods about the $\alpha_{k}, k=1, \cdots, g$, and the $\Gamma_{\nu}, \nu=1, \cdots, n$. $p(z, \zeta)$ is easily constructed by first adding to the analytic function whose real part is the Green's function of $R$ a suitable linear combination, with imaginary coefficients, of functions $w_{k}^{*}(z)$ which removes its periods about the $\alpha_{k}$, and then adding another linear combination of functions $W_{\nu}(z)$, with real coefficients, which makes the periods about the $\Gamma_{\nu}$ vanish.

The second type of function of the third kind cannot be defined by merely substituting constancy of the imaginary part on $\Gamma_{\nu}$ for constancy of the real part in the above definition. In fact, it is easy to show that, even in the case $g=0$, such a function does not exist. This difficulty can, however, be circumvented, by introducing a function of three arguments, $q(z ; u, v)$, which possesses the following properties. $q=q(z ; u, v)$ is regular in $R$, except at $z=u$ and $z=v$, where $q+\log (z-u)$ and $q-\log (z-v)$, respectively, are regular; the imaginary part of $q$ is constant on the boundary components $\Gamma_{\nu}$ and $q$ has no periods about the $\alpha_{k}$ and the $\Gamma_{\nu}$.

The construction of $q(z ; u, v)$ proceeds as follows. Let $N(z, u)$ be the Neumann's function of $R$, that is, the function which is harmonic and singlevalued in $R$, except at $z=u$, where $N(z, u)+\log |z-u|$ is harmonic, and has, on $\Gamma$, the constant normal derivative $2 \pi / L$, where $L$ is the length of $\Gamma$. Let $\tilde{N}(z, u)$ denote the harmonic conjugate of $N(z, u)$, and consider the expression

$$
P=N(z, u)+i \tilde{N}(z, u)-N(z, v)-i \tilde{N}(z, v) .
$$

Since, for $z \in \Gamma$,

$$
d \tilde{N}=\frac{\partial \tilde{N}}{\partial s} d s=\frac{\partial N}{\partial n} d s=\frac{2 \pi}{L} d s
$$

we have

$$
d P=d[N(z, u)-N(z, v)]
$$

on $\Gamma$, that is, the imaginary part of $P$ is constant on each $\Gamma_{\nu}$. We now add to $P$ a linear combination, with imaginary coefficients, of functions $w_{k}(z)$ which cancels the periods of $P$ about the $\alpha_{k}$, and finally add a linear combination, with imaginary coefficients, of functions $W_{\nu}(z)$ which removes the periods 
about the $\Gamma_{\boldsymbol{\nu}}$. The function which we obtain in this way is easily identified with the desired function of the third kind $q(z ; u, v)$.

In order to obtain complete symmetry in the definitions of the two types of functions of the third kind, it is advisable to define also the first type of these functions as a function of three arguments. We write

$$
p(z ; u, v)=p(z, u)-p(z, v) .
$$

9. Periods of the functions of the third kind. The periods of the functions $p$ and $q$ about the $\beta_{k}$ are computed in a similar fashion as those of the functions of the second kind. When $\Gamma^{*}$ again denotes the boundary of the domain $R^{*}$ into which $R$ is transformed by a system of canonical cuts, we have, by the residue theorem,

$$
\frac{1}{2 \pi i} \int_{\Gamma^{*}} p^{\prime}(z, \zeta) w_{k}(z) d z=w_{k}(\zeta)
$$

As in $\S 4$, the integrals over the $\alpha_{l}$ and $\beta_{l}$ cancel out, with the exception of the integrals over both edges of $\beta_{k}$ which contribute a term $(1 / 2 \pi i) \int_{\beta_{k}} p^{\prime}(z, \zeta) d z$. We thus have

$$
w_{k}(\zeta)=\frac{1}{2 \pi i} \int_{\beta_{k}} p^{\prime}(z, \zeta) d z+\sum_{\nu=1}^{n} \frac{1}{2 \pi i} \int_{\Gamma_{\nu}} p^{\prime}(z, \zeta) w_{k}(z) d z .
$$

On the $\Gamma_{\nu}, p^{\prime}(z, \zeta) d z=d p(z, \zeta)$ is pure imaginary, and so is $w_{k}(z)$. Taking real parts, we therefore obtain

$$
\operatorname{Re}\left\{w_{k}(\zeta)\right\}=\operatorname{Re}\left\{\frac{1}{2 \pi i} \int_{\beta_{k}} p^{\prime}(z, \zeta) d z\right\} .
$$

Replacing $w_{k}(z)$ by $w_{k}^{*}(z)$, we obtain in a similar fashion

$$
\operatorname{Im}\left\{w_{k}^{*}(\zeta)\right\}=\operatorname{Im}\left\{\frac{1}{2 \pi i} \int_{\beta_{k}} p^{\prime}(z, \zeta) d z\right\}
$$

and finally,

$$
\frac{1}{2 \pi i} \int_{\beta_{k}} p^{\prime}(z, \zeta) d z=\operatorname{Re}\left\{w_{k}(\zeta)\right\}+i\left\{\operatorname{Im} w_{k}^{*}(\zeta)\right\}
$$

For the function of three variables, $p(z ; u, v)$, we have, by $(27)$,

(29) $\frac{1}{2 \pi i} \int_{\beta_{k}} p^{\prime}(z ; u, v) d z=\operatorname{Re}\left\{w_{k}(u)-w_{k}(v)\right\}+i \operatorname{Im}\left\{w_{k}^{*}(u)-w_{k}^{*}(v)\right\}$, and for $q(z ; u, v)$, we obtain, by the same procedure as before, (30) $\frac{1}{2 \pi i} \int_{\beta_{k}} q^{\prime}(z ; u, v) d z=\operatorname{Re}\left\{w_{k}^{*}(u)-w_{k}^{*}(v)\right\}+i \operatorname{Im}\left\{w_{k}(u)-w_{k}(v)\right\}$. 
10. Circular and radial slit mappings. Having defined the functions of the third kind on an open Riemann surface and having expressed their periods in terms of functions of the first kind, we are now in a position to prove the following mapping theorem.

ThEOREM. Every open Riemann surface $R$ of genus $g$ can be mapped conformally onto another open Riemann surface which has no more than $g+1$ sheets and is bounded by slits along circular arcs centered at the origin; we can moreover prescribe the location of one pole and one zero of the mapping function. The same statement holds if the circular slits are replaced by rectilinear slits pointing at the origin.

Proof. We shall confine ourselves to the case of the circular slit mapping. The radial slit mapping is proved in a very similar manner, the only major difference being that the function $p(z ; u, v)$ we shall use in the following argument is to be replaced by $q(z ; u, v)$.

Suppose that $z=u_{0}$ is the given zero and $z=v_{0}$ is the given pole of the mapping function, and consider the function

$$
g(z)=\sum_{\mu=0}^{g} p\left(z ; u_{\mu}, v_{\mu}\right)
$$

where $p(z ; u, v)$ is the function of the third kind introduced above and $u_{\mu}, v_{\mu}, \mu=1, \cdots, g$, are points of $R$. By (29), $g(z)$ will have-up to the common factor $2 \pi i$-the period

$$
\lambda_{k}=\operatorname{Re}\left\{\sum_{\mu=0}^{g} w_{k}\left(u_{\mu}\right)-w_{k}\left(v_{\mu}\right)\right\}+i \operatorname{Im}\left\{\sum_{\mu=0}^{g} w_{k}^{*}\left(u_{\mu}\right)-w_{k}^{*}\left(v_{\mu}\right)\right\}
$$

about the path $\beta_{k}, k=1, \cdots, g$. Apart from the $\lambda_{k}$, the only periods of $g(z)$ are those caused by the logarithmic poles.

Denote by $a_{k}$ and $b_{k}$ the expressions

$$
a_{k}=-\operatorname{Re}\left\{w_{k}\left(u_{0}\right)-w_{k}\left(v_{0}\right)\right\}, \quad b_{k}=-\operatorname{Im}\left\{w^{*}\left(u_{0}\right)-w^{*}\left(v_{0}\right)\right\},
$$

and suppose that the points $u_{\mu}$ and $v_{\mu}, \mu=1, \cdots, g$, can be chosen so that the equations

$$
\begin{aligned}
& \operatorname{Re}\left\{\sum_{\mu=1}^{g} w_{k}\left(u_{\mu}\right)-w_{k}\left(v_{\mu}\right)\right\}=a_{k}, \quad k=1, \cdots, g, \\
& \operatorname{Im}\left\{\sum_{\mu=1}^{g} w_{k}^{*}\left(u_{\mu}\right)-w_{k}\left(v_{\mu}\right)\right\}=b_{k}
\end{aligned}
$$

are satisfied. In view of (32), this entails the vanishing of the periods $\lambda_{k}$ of $g(z)$. The only periods such a function $g(z)$ will have will therefore be the periods $2 \pi i$ about sufficiently small paths surrounding the points $u_{\mu}, \mu$ 
$=0,1, \cdots, g$, and, similarly, the periods $-2 \pi i$ about the points $v_{\mu}$. The function $f(z)=e^{o(z)}$ will therefore be single-valued in $R$ and have simple poles at the points $v_{\mu}, \mu=0,1, \cdots, g$, and simple zeros at the points $u_{\mu}$. The real parts of the functions $p(z ; u, v)$ are constant on the boundary components $\Gamma_{\nu}, \nu=1, \cdots, n$. By (31), the same is true of $g(z)$. In view of $d|f| /|f|$ $=d \operatorname{Re}\{g\}$, we have therefore

$$
d|f|=0, \quad z \in \Gamma_{\nu}, \quad \nu=1, \cdots, n,
$$

that is, the modulus of $f(z)$ is constant on each boundary component. Since the function $g(z)$ has no periods about the $\Gamma_{\nu}$, the variation of $\arg f(z)$ along each $\Gamma_{\nu}$ vanishes. Using the argument principle, it is therefore easily shown that $w=f(z)$ maps $R$ onto an open Riemann surface consisting of $g+1$ sheets, all covering the full w-plane, whose boundaries are $n$ circular slits centered at the origin.

Our theorem will therefore be proved if we can show that, for arbitrarily given $a_{k}, b_{k}$, it is always possible to find $2 g$ points $u_{\mu}, v_{\mu}, \mu=1, \cdots, g$, on $R$ so that the system of equations (33) is satisfied.

It is clearly sufficient to prove this possibility for any open Riemann surface which is conformally equivalent to $R$. As such we shall choose the surface $A$ obtained from $R$ by the Ahlfors mapping described in $\$ 1$ and a subsequent linear substitution transforming the interior of the unit circle into the upper half-plane. This surface $A$ consists of not more than $n+2 g$ sheets, all covering the entire upper half-plane; all boundary components of $A$ lie on the real axis.

The mirror image of $A$ with respect to the real axis will be denoted by $\bar{A}$. By connecting $A$ with $\bar{A}$ along their corresponding boundary components we obtain a closed Riemann surface, which we denote by $B$. By elementary considerations, the genus of $B$ is found to be $p=2 g+n-1$.

The function of the first kind on the open surface $A$-which we again denote by $w_{k}(z)$ and $w_{k}^{*}(z)$-have particularly simple properties. Since $w_{k}(z)$ is pure imaginary on $\Gamma$ and $w_{k}^{*}(z)$ is real there and, on the other hand, $\Gamma$ coincides with the real axis, both $w_{k}(z)$ and $w_{k}^{*}(z)$ can be analytically continued-by Schwarz' reflection principle-throughout the whole closed surface $B$ by means of the equations

$$
w(\bar{z})=-\overline{w(z)}, \quad w^{*}(z)=\overline{w^{*}(z)} .
$$

In view of (34), the system (33) may be replaced by

$$
\begin{aligned}
& \operatorname{Re}\left\{\sum_{\mu=1}^{g} w_{k}\left(u_{\mu}\right)+w_{k}\left(\bar{v}_{\mu}\right)\right\}=a_{k}, \quad k=1, \cdots, g, \\
& \operatorname{Im}\left\{\sum_{\mu=1}^{g} w_{k}^{*}\left(u_{\mu}\right)+w_{k}^{*}\left(\bar{v}_{\mu}\right)\right\}=b_{k} .
\end{aligned}
$$


We now choose a system of $p(p=2 g+n-1)$ pairs of canonical cuts $\alpha_{\sigma}^{(B)}$, $\beta_{\sigma}^{(B)}, \sigma=1, \cdots, p$, on $B$. Obviously, $g$ of the curves $\alpha_{\sigma}^{(B)}$, say for $\sigma=1, \cdots, g$, may be taken to coincide with the curves $\alpha_{1}, \cdots, \alpha_{g}$ on $A$, and a further set of $g$ of the curves $\alpha_{\sigma}^{(B)}$, say for $\sigma=g+1, \cdots, 2 g$, may be identified-apart from their orientation-with the mirror images of the $\alpha_{k}, k=1, \cdots, g$.

Since the remaining $n-1$ pairs of periods drop out when $B$ is separated into $A$ and $\bar{A}$, we may identify the balance of $n-1$ curves $\alpha_{\sigma}^{(B)}$ with the $n-1$ closed curves obtained by describing $n-1$ of the $n$ boundary components $\Gamma_{\nu}$ back and forth. We denote by $V_{\sigma}(z), \sigma=1, \cdots, p$, the normal Abelian integrals of the first kind on $B$ related to our particular choice of the $\alpha_{\sigma}^{(B)}$. By its definition, the function $V_{\sigma}(z)$ is free of singularities on $B$, has the period 1 about $\alpha_{\sigma}^{(B)}$, and has no periods about $\alpha_{\tau}^{(B)}, \tau \neq \sigma$.

Consider now the function

$$
\overline{V_{k}(\bar{z})}, \quad k=1, \cdots, g .
$$

In view of the symmetry of $B$, this function will also be free of singularities in $B$. It will clearly be free of periods about all the $\alpha_{\sigma}^{(B)}$ with the exception of $\alpha_{k+g}^{(B)}$, about which its period will be -1 (since the orientation of the surface has been changed by the reflection). Hence,

$$
\overline{V_{k}(\bar{z})}=-V_{o+k}(z), \quad k=1, \cdots, g .
$$

Accordingly, the function $V_{k}(z)+V_{o+k}(z)$ will be pure imaginary if $z$ is on the real axis. Indeed,

$$
V_{k}(z)+V_{g+k}(z)=V_{k}(z)-\overline{V_{k}(\bar{z})}=V_{k}(z)-\overline{V_{k}(z)}=i \operatorname{Im}\left\{V_{k}(z)\right\} \quad(z=\bar{z}) .
$$

Since, moreover, this function has no periods about the $\Gamma_{\nu}$ and the $\alpha_{l}(l \neq k)$, and the period 1 about $\alpha_{k}$, it is identical with the function of the first kind $w_{k}(z)$ on $A$. Thus,

$$
w_{k}(z)=V_{k}(z)+V_{o+k}(z) .
$$

Similarly, we obtain from (36) the identity

$$
w_{k}^{*}(z)=V_{k}(z)-V_{o+k}(z) .
$$

With the help of these relations we shall now show that-apart from some additional conditions which have to be dealt with separately-the solution of the system (35) can be reduced to the classical Jacobi inversion problem related to the closed Riemann surface $B$. In our case, the Jacobi problem consists in finding a set of $p$ points $t_{\tau}, \tau=1, \cdots, p$, on $B$ such that the equations

$$
\sum_{\tau=1}^{p} V_{\sigma}\left(z_{\tau}\right)=\gamma_{\sigma}, \quad \sigma=1, \cdots, p
$$


are satisfied, where the $\gamma_{\sigma}$ are arbitrarily given complex numbers, not all zero. It was shown by Riemann [10] and Weierstrass [12] that the Jacobi problem always has a solution and that the $z_{\tau}$ are analytic functions of the arguments $\gamma_{1}, \cdots, \gamma_{p}$.

Adding and subtracting the rows of index $k$ and $k+g(k=1, \cdots, g)$ in (39), we see, in view of (37) and (38), that (39) can be replaced by

$$
\begin{array}{lr}
\sum_{\tau=1}^{p} w_{k}\left(z_{\tau}\right)=a_{k}+i a_{k}{ }^{\prime}, & k=1, \cdots, g, \\
\sum_{\tau=1}^{p} w_{k}^{*}\left(z_{\tau}\right)=b_{k}{ }^{\prime}+i b_{k}, & \\
\sum_{\tau=1}^{p} V_{2 g+\nu}\left(z_{\tau}\right)=\gamma_{2 o+\nu}, & \nu=1, \cdots, n-1,
\end{array}
$$

where $a_{k}+i a_{k}^{\prime}, \quad b_{k}{ }^{\prime}+i b_{k}\left(a_{k}, a_{k}^{\prime}, b_{k}, \quad b_{k}^{\prime}\right.$ real $)$ has been written instead of $\gamma_{k}+\gamma_{o+k}$ and $\gamma_{k}-\gamma_{o+k}$. In view of the solvability of the Jacobi problem, the system (40) can therefore be solved for arbitrarily given values of the constants on the right-hand side.

Taking the real part of the first equation (40) and the imaginary part of the second one, we obtain

$$
\operatorname{Re}\left\{\sum_{\tau=1}^{p} w_{k}\left(z_{\tau}\right)\right\}=a_{k}, \quad \operatorname{Im}\left\{\sum_{\tau=1}^{p} w_{k}^{*}\left(z_{\tau}\right)\right\}=b_{k} .
$$

This system would be identical with the system (35) upon the solvability of which the proof of our theorem devolves, if $n-1$ of the points $z$ were situated on the real axis and, of the remaining $2 g$ points, $g$ points were in the upper and $g$ points in the lower half-plane. Indeed, $\operatorname{Re}\left\{w_{k}(z)\right\}=\operatorname{Im}\left\{w_{k}^{*}(z)\right\}=0$ for real $z$, and the $g$ points $z_{\tau}$ in the lower half-plane can be identified with the point $\bar{v}_{\mu}$ in (35). Our task is therefore reduced to showing that by giving suitable values to the-so far arbitrary-constants $a_{k}^{\prime}, b_{k}^{\prime}, \gamma_{20+\nu}$ in (40) we can obtain a solution $z_{1}, \cdots, z_{p}$ of this system of equations which has this particular distribution.

The Jacobi problem (40) always has a solution and, as already mentioned, the solving points $z$ are analytic functions of the parameters on the righthand side. We now apply small variations $\delta a_{k}^{\prime}, \delta b_{k}^{\prime}, \delta s_{\nu}, \delta t_{\nu}\left(\gamma_{20+\nu}=s_{\nu}+i t_{\nu}\right)$ to the arbitrary parameters on the right-hand side. This leads us to a solution $z_{\tau}+i \delta z_{\tau}$ of a slightly modified Jacobi problem, which will still satisfy the equations (41). $\mathrm{Up}$ to second-order terms, the variations of the parameters and the variation $\delta z_{\tau}$ will be related by

$$
\sum_{\tau=1}^{p} w_{k}^{\prime}\left(z_{\tau}\right) \delta z_{\tau}=i \delta a_{k}^{\prime}, \quad \sum_{\tau=1}^{p} w_{k}^{* \prime}\left(z_{\tau}\right) \delta z_{\tau}=\delta b_{k}^{\prime}, \quad \sum_{\tau=1}^{p} V_{2 g+\nu}^{\prime}\left(z_{\tau}\right) \delta z_{\tau}=\delta s_{\nu}+i \delta t_{\nu} .
$$


Using the notations

$$
\begin{aligned}
w_{k}^{\prime}\left(z_{\tau}\right) & =c_{k \tau}+i c_{k \tau}^{\prime}, & w_{k}^{*}\left(z_{\tau}\right) & =c_{k \tau}^{*}+i c_{k \tau}^{* \prime}, \\
V_{2 \sigma+\nu}^{\prime}\left(z_{\tau}\right) & =d_{v \tau}+i d_{\nu \tau}^{\prime}, & z_{\tau} & =x_{\tau}+i y_{\tau},
\end{aligned}
$$

and separating real and imaginary parts, this may also be written

$$
\begin{aligned}
& \sum_{\tau=1}^{p} c_{k \tau} \delta x_{\tau}-c_{k \tau}^{\prime} \delta y_{\tau}=0, \\
& \sum_{\tau=1}^{p} c_{k \tau}^{\prime} \delta x_{\tau}+c_{k \tau} \delta y_{\tau}=\delta a_{k}^{\prime}, \quad k=1, \cdots, g, \\
& \sum_{\tau=1}^{p}{ }_{c_{k \tau}^{*} \delta x_{\tau}-c_{k \tau}^{* \prime} \delta y_{\tau}}^{*}=\delta b_{k}^{\prime} ; \\
& \sum_{\tau=1}^{p}{ }_{c_{k \tau}^{* \prime} \delta x_{\tau}+c_{k \tau}^{*} \delta y_{\tau}}^{*}=0, \\
& \sum_{\tau=1}^{p} d_{\nu \tau} \delta x_{\tau}-d_{\nu \tau}^{\prime} \delta y_{\tau}=\delta s_{\nu}, \\
& \sum_{\tau=1}^{p} d_{\nu \tau}^{\prime} \delta x_{\tau}+d_{\nu \tau} \delta y_{\tau}=\delta t_{\nu} .
\end{aligned}
$$

If we could show that, for arbitrarily given variations $\delta y_{\tau}$, we can always determine corresponding variations $\delta x_{\tau}, \delta a_{k}{ }^{\prime}, \delta b_{k}{ }^{\prime}, \delta t_{\nu}, \delta s_{\nu}$, so that this system of equations is satisfied, we would have proved that the solutions $z_{\tau}$ can be arbitrarily moved "up and down" without violating the conditions (41). This, in turn, would show that we can always obtain the desired configuration of solutions $z_{\tau}$, namely, $g$ points with $\operatorname{Im}\left\{z_{\tau}\right\}>0, g$ points with $\operatorname{Im}\left\{z_{\tau}\right\}<0$, and $n-1$ points satisfying $\operatorname{Im}\left\{z_{\tau}\right\}=0$.

Let now $\delta y_{\tau}, \tau=1, \cdots, p$, be an arbitrary "vertical" variation. If the rank of the matrix

$$
M=\left(\begin{array}{ccc}
c_{11} & \cdots & c_{1 p} \\
\cdot & \cdot & \cdot \\
c_{g 1} & \cdots & c_{g p} \\
c_{11}^{* \prime} & \cdots & c_{1 p}^{* \prime} \\
\cdot & \cdots & \cdot \\
c_{01}^{* \prime} & \cdots & c_{g p}^{* \prime}
\end{array}\right)
$$

is $2 g$, there exists a system of solutions $\delta x_{1}, \cdots, \delta x_{2 \sigma}$ of the equations (43) and (44). Inserting these solutions in the equations below (43) and (44), these equations can then be satisfied by giving to $\delta a_{k}^{\prime}, \delta b_{k}^{\prime}, \delta s_{\nu}, \delta t_{\nu}$ suitable values. 
As long as the rank of the matrix $M$ is $2 g$, the ordinates of the points $z_{\tau}$ can therefore be arbitrarily changed in a continuous manner, without these points ceasing to be solutions of (41).

This argument fails, however, if the rank of $M$ is lower than $2 g$. In this case we proceed as follows. We set $\delta y_{\tau}=0, \tau=1, \cdots, p$, and try to find a nontrivial solution $\delta x_{\tau}, \tau=1, \cdots, p$, of the system (43) and (44). Such a solution certainly exists, since, in view of the fact that the rank of $M$ is lower than $2 g$, the homogeneous equations

$$
\begin{aligned}
& \sum_{\tau=1}^{p} c_{k \tau} \delta x_{\tau}=0, \quad k=1, \cdots, g, \\
& \sum_{\tau=1}^{p} c_{k \tau}^{* \prime} \delta x_{\tau}=0
\end{aligned}
$$

have a nontrivial solution. There exists, therefore, in this case, a "horizontal" variation $\delta z_{\tau}$ which leaves the conditions (41) unchanged. Now there are two possibilities. Either the matrix $M$ belonging to the points $z_{\tau}+x_{\tau}$ is already of rank $2 g$, or else there are finite horizontal intervals adjacent to one or more of the points $z_{\tau}$ in which the rank of $M$ is still lower than $2 g$. If the first alternative is true, we are back in the case discussed before and we may continue to apply "vertical" variations. All that remains to discuss, therefore, is the second alternative.

In that case, all determinants containing $2 g$ columns of $M$ vanish identically if at least one of the variables $z_{\tau}$, say $z_{1}$, on which it depends varies in a finite horizontal interval. Keeping the other variables constant, the vanishing of the determinant is equivalent to the identity

$$
P(z)=\sum_{k=1}^{g}\left[h_{k} \operatorname{Re}\left\{w_{k}^{\prime}(z)\right\}+h_{k}^{\prime} \operatorname{Im}\left\{w_{k}^{* \prime}(z)\right\}\right]=0, \quad h_{k}, h_{k}^{\prime}=\text { const. }
$$

if $z$ varies in a finite horizontal interval. Since (45) is everywhere in $B$ a regular harmonic function, (45) is even true on the whole horizontal line $L$ of which the interval in question forms part. Beyond $L, P(z)$ can be continued by the Schwarz inversion principle, $P(z)$ being of the same absolute value but of different sign at two points symmetric to $L$. On the other hand, we have also $P(z) \equiv 0$ on the real axis, since there $w_{k}^{\prime}(z)$ is imaginary and $w_{k}^{* \prime}(z)$ real. If $L$ were different from the real axis, the function $P(z)$ would therefore have the periodic property $P(z+2 d i)=P(z)$, where $d$ is the distance of $L$ from the real axis, which is absurd.

We have thus proved that the point $z_{1}$ lies on the real axis. Since each of the determinants of order $2 g$ to be formed from $M$ depends only on $2 g$ points $z_{r}$ and excludes $(n-1)$ other points, and the preceding argument is valid for every such determinant, it follows that, in the case under discussion, at least $n$ of the points $z_{\tau}$ must lie on the real axis. 
Summing up our results, we have shown that it is always possible to make arbitrary continuous variations of the ordinates of the points $z_{\tau}$, $\tau=1, \cdots, p$, without violating the conditions (41), provided not more than $n-1$ points $z_{\tau}$ are located on the real axis at the same time. In particular, it is always possible to obtain such solutions of (41) for which $g$ of the points $z_{r}$ are in the upper half-plane, $g$ others are in the lower half-plane, and the remaining $n-1$ points lie on the real axis. As shown before, this implies the proof of our mapping theorem. It should be noted that these circular slit mappings are not unique. As shown by the proof, there is a family depending on $2 g$ real parameters of such mappings. Only for $g=0$, that is, in the schlicht case, is the mapping uniquely determined.

\section{BIBLIOGRAPHY}

1. L. Ahlfors, Material presented in a colloquium lecture at Harvard University in Spring 1948.

2. S. Bergman, Ueber die Entwicklung der harmonischen Funktionen der Ebene und des Raumes nach Orthogonalfunktionen, Math. Ann. vol. 86 (1926) pp. 238-271.

3. - Ueber die Kernfunktion eines Bereiches und ihr Verhalten am Rande, J. Reine Angew. Math. vol. 169 (1932) pp. 1-42.

4. - - Sur les fonctions orthogonales de plusieurs variables complexes, Mémorial des Sciences Mathématiques, vol. 106, 1947.

5. L. Bieberbach, Ueber einen Riemannschen Satz aus der Lehre von der konformen Abbildung, Berlin Math. Ges. Sitzungsber. vol. 24 (1925) pp. 6-9.

6. P. Garabedian and M. Schiffer, Identities in the theory of conformal mapping, Trans. Amer. Math. Soc. vol. 65 (1949) pp. 187-238.

7. H. Grunsky, Ueber die konforme Abbildung mehrfach zusammenhaengender Bereiche auf mehrblaettrige Kreise, I., Preuss. Akad. Wiss. Sitzungsber., 1937, pp. 40-46, II. Abhandlungen der Preussischen Akademie der Wissenschaften, Mathematisch-Naturwissenschaftliche Klasse 1941, no. 11.

8. - Neue Abschaetzungen zur konformen Abbildung ein- und mehrfach zusammenhaengender Bereiche, Schriften des Mathematischen Seminars der Universität Berlin vol. 1 (1932) pp. 94-140.

9. Z. Nehari, The kernel function and canonical conformal maps, Duke Math. J. vol. 16 (1949) pp. 165-178.

10. B. Riemann, Werke, 2d ed., pp. 88-142; pp. 212-224.

11. E. Schottky, Ueber die konforme Abbildung mehrfach zusammenhaengender ebener Flaechen, J. Reine Angew. Math. vol. 83 (1877) pp. 300-351.

12. C. Weierstrass, Vorlesungen ueber die Theorie der Abelschen Transzendenten, Werke, vol. 4 .

HARVARD University,

Cambridge, Mass.

WASHINGTON UNIVERSITY, St. Louis, Mo. 